\title{
Vorwort des Herausgebers
}

Wie viele Publikationen des Forschungsarchivs für Antike Plastik, so hat auch dieser Katalog eine längere Vorgeschichte. Bereits in den 1980er Jahren war Andreas Linfert, Professor für Klassische Archäologie der Universität zu Köln, während seiner Forschungsreisen auf die Sammlung antiker Skulpturen in Laon aufmerksam geworden. In enger Abstimmung mit dem Museum und mit weiteren französischen Kollegen entwickelte er das Projekt eines Katalogs. Aus Mitteln des Forschungsarchivs sowie mit finanzieller Unterstützung der Freunde und Förderer der Universität zu Köln konnte 1994 mit der Fotomeisterin Gisela Geng eine Fotokampagne durchgeführt werden, mit der die Stücke in qualitativ hochstehenden Aufnahmen dokumentiert wurden.

Der frühe Tod Andreas Linferts im Jahre 1996 verhinderte den Abschluss und die Publikation des Katalogs. ${ }^{1}$ Als Paul Scheding 2013 vorübergehend die Leitung des Forschungsarchivs übernahm, griff er das Projekt einer Vorlage der antiken Skulpturen in Laon erneut auf. Dabei zeigte sich, dass die vorliegenden provisorischen Texte für eine Publikation nicht mehr geeignet waren. Zum einen ergab sich für zahlreiche Stücke aufgrund der neuen Forschungslage eine veränderte Einschätzung; zum anderen erlaubte die inzwischen erfolgte Erschließung unpublizierter Archivunterlagen neue Erkenntnisse über Provenienzen sowie über die Anliegen und Urteile des Sammlers.

Ein Stipendium der Fritz Thyssen Stiftung für Karolina Kaderka von der École Pratique des Hautes Études in Paris ermöglichte die grundlegende Neubearbeitung der Samm- lung und erbrachte neue Kenntnisse zu ihrer Geschichte. Paul Scheding, der inzwischen eine Stelle als Akademischer Rat an der Ludwig-Maximilians-Universität in München angetreten hatte, übernahm die zweite Hälfte der Katalogbeiträge. Philipp Groß und Lisa Schadow vom Forschungsarchiv für antike Plastik bereiteten die Abbildungsvorlagen für den Druck vor. Anne Kleineberg half bei der Korrektur der Fahnen. François Queyrel (École Pratique des Hautes Études, Paris) und Christiane Vorster (Universität Bonn), beide durch ihre Publikationen zur antiken Plastik international renommiert, diskutierten mit den Autoren bei zwei von der Fritz Thyssen Stiftung finanzierten Treffen im Museum von Laon die Ergebnisse und lasen die hier vorliegenden Texte.

Entscheidend für das Gelingen des Projekts war die Unterstützung durch das Musée de Laon, insbesondere durch den Direktor Rémi Bazin, seine Mitarbeiterin Morgane Reck und die langjährige Kuratorin Caroline Jorrand. Sie ermöglichten den Zugang zu den Objekten in den Magazinen und in den Ausstellungsräumen, halfen bei der Untersuchung der Stücke, öffneten großzügig die Archive für das Studium der Sammlungsgeschichte und lösten die zahlreichen technischen Schwierigkeiten, die im Verlauf der Arbeiten auftraten.

Mein Dank gilt insbesondere der Fritz Thyssen Stiftung, die durch ihre Förderung das Projekt möglich gemacht hat; zudem dem Reichert Verlag und Miriam Würfel für die intensive und freundschaftliche Zusammenarbeit.

Köln, im Mai 2021

Dietrich Boschung 


\section{Avant-Propos}

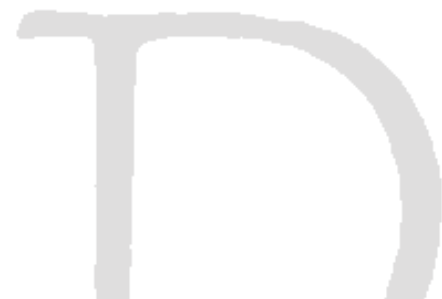

C’est un fait, le Musée du Pays de Laon est connu des spécialistes pour la richesse de ses collections archéologiques. Nous y trouvons naturellement d'innombrables vestiges d'histoire régionale, de la Préhistoire jusqu'au Moyen Âge. Plus surprenante est la collection d'antiquités méditerranéennes accueillie au musée en 1937, léguée par un industriel laonnois, helléniste distingué et collectionneur avisé, Paul Marguerite de la Charlonie. La Grande Guerre puis son décès, en 1921, ont eu raison de sa grande ambition : la création à Paris d'un musée de l'Hellénisme. Restent la quantité et la qualité impressionnantes des objets regroupés dans ce que nous appelons communément - non sans tendresse et beaucoup d'admiration - la « collection La Charlonie». Complétée par quelques éléments provenant de la collection Campana, elle constitue un ensemble exceptionnel qui participe au rayonnement du Musée d'art et d'archéologie du Pays de Laon.

Présentée à partir des années 1950, cette collection attire l'attention des spécialistes français et étrangers, mais ce sont principalement les céramiques, vases à décor et figurines, qui font l'objet d'études et de publications. Bien peu de chercheurs s'intéressent aux éléments sculptés que l'on trouve également dans cette collection. C'est le cas d'Andreas Linfert, professeur à l'Archäologisches Institut de l'Université de Cologne, qui prend contact avec le musée de Laon en vue de leur étude. En 1984, il écrit à M. Marcadé, professeur d'archéologie classique à l'Université de Paris I : « pourquoi les français ne se sont-ils intéressés qu'aux vases ? " Là est toute la question... Son projet d'étude se transforme vite en projet d'édition d'un Inventaire des sculptures grecques et romaines du musée archéologique de Laon. Envisagé avec le département Éditions de la Réunion des Musées Nationaux puis avec un éditeur allemand, ce projet achoppe sur la question de son financement. Après douze ans de négociations infructueuses, le décès d'Andreas Linfert en 1996 met un terme à ce beau projet.

Mais l'ensemble des sculptures des collections La Charlonie et Campana se devait d'avoir la reconnaissance scientifique qu'il mérite et c'est avec une immense satisfaction que le Musée du Pays de Laon a accueilli ce nouveau projet d'édition mené conjointement par les Universités de Cologne, Bonn et Munich ainsi que l'École Pratique des Hautes Études au sein de l'Université Paris Sciences \& Lettres. C'est un honneur de susciter l'intérêt des chercheurs de ces établissements prestigieux et, certainement, une chance exceptionnelle qui nous est donnée de mieux connaître et mettre en valeur cette magnifique collection, dans la globalité comme dans le détail. C'est également, une fois encore, l'occasion d'une belle rencontre avec des historiens passionnés qui font vivre nos merveilleuses antiquités.

\section{Rémi Bazin}

Directeur du Musée d'art et d'archéologie du Pays de Laon 